\title{
Enhanced Expression of Transgenes from Adeno-Associated Virus Vectors with the Woodchuck Hepatitis Virus Posttranscriptional Regulatory Element: Implications for Gene Therapy
}

\author{
JONATHAN E. LOEB, ${ }^{1,2}$ WENDY S. CORDIER, ${ }^{3}$ MATTHEW E. HARRIS, ${ }^{1}$ \\ MATTHEW D. WEITZMAN, ${ }^{3}$ and THOMAS J. HOPE ${ }^{1}$
}

\begin{abstract}
The woodchuck hepatitis virus posttranscriptional regulatory element (WPRE) evolved to stimulate the expression of intronless viral messages. To determine whether this ability to enhance expression could be useful in nonviral and heterologous viral gene delivery systems, we analyzed the ability of the WPRE to elevate the expression of a cDNA encoding the green fluorescent protein (GFP) in these contexts. We find that the WPRE can stimulate the expression of GFP when the gene is delivered by transfection or transduction with recombinant adeno-associated virus (AAV). Enhancement occurred both during transient expression and when the gene is stably incorporated into the genome of target cells. This enhancement required that the WPRE be located in cis within the GFP message, and was observed in both transformed cell lines and primary human fibroblasts. These results demonstrate that the WPRE will be an effective tool for increasing the long-term expression of transgenes in gene therapy.
\end{abstract}

\section{OVERVIEW SUMMARY}

The optimization of transgene expression at the posttranscriptional level will enhance the capacity of vector delivery systems to produce therapeutic results in target tissues. In this study, the woodchuck hepatitis virus posttranscrip tional regulatory element (WPRE) is shown to enhance the expression of an eGFP reporter gene in both transient and stable contexts, when delivered either by transfection or by transduction with recombinant $\mathrm{AAV}$. This enhancement of transgene expression is observed both in cell lines and in primary cells. Inclusion of the WPRE in transgenic messages will better the prospects for therapeutic levels of transgene expression using currently existing gene delivery technologies.

\section{INTRODUCTION}

$\mathbf{T}$ He effective Deliver y of transgenes to target cells for therapeutic purposes will require strategies that optimize this process at each step. Previous efforts at enhancing transgene expression have largely been directed at improving efficiency of delivery and increasing levels of transcription. Gene expression can be modulated at many levels, including transcription, posttranscriptional modification of RNA such as $5^{\prime}$ and $3^{\prime}$ end processing, RNA export and stability, and translation. Understanding of the mechanism s by which viruses posttranscriptionally modulate expression of their genes should enable the exploitation of these mechanisms for the purpose of improving expression in gene therapy. Attempts at posttranscriptional enhancement have primarily been limited to the addition of in-

\footnotetext{
${ }^{1}$ Infectious Disease Laboratory, The Salk Institute for Biological Studies, La Jolla, CA 92037.

${ }^{2}$ Biomedical Sciences Graduate Program, University of California, San Diego, La Jolla, CA 92093.

${ }^{3}$ Laboratory of Genetics, The Salk Institute for Biological Studies, La Jolla, CA 92037.
} 
trons to the $3^{\prime}$ end of the RNA of interest (Choi et al., 1991; Wang et al., 1996). In these reports, the inclusion of introns was shown to stimulate gene expression. Although the exact mechanism of this stimulation is not known, intronic sequences, or the process of splicing itself, may promote $3^{\prime}$ processing and/or facilitate cytoplasmic localization (Ryu and Mertz, 1989; Nesic et al., 1993; Antoniou et al., 1998). Indeed, certain genes, such as $\beta$-globin, are entirely dependent on the presence of introns for expression (Buchman and Berg, 1988).

It has previously been shown that the posttranscriptiona 1 regulatory element of hepatitis B virus (HPRE) can functionally replace an intron in stimulating $\beta$-globin expression (Huang and Yen, 1995). Our studies of the woodchuck hepatitis virus demonstrated that it contains a posttranscriptiona 1 regulatory element (WPRE) that is partially homologous to the HPRE (Donello et al., 1998). Both PREs function in an orientationdependent manner, suggesting that they are RNA elements. Consistent with this interpretation, the PREs contain conserved RNA stem-loop structures that are required for maximal function (Smith et al., 1998). We find that the WPRE functions more efficiently than the HPRE, owing to the presence of an additional cis-acting sequence in the WPRE termed $\gamma$ (Donello et al., 1998). The specific mechanism of this enhancement, while not well understood, is known to be posttranscriptional in nature and may involve the facilitation of RNA processing and/or export (Harris et al., 1999; Huang et al., 1999).

Due to the high efficiency of the WPRE, we assayed the effectiveness of the WPRE as a means of enhancing gene expression in the context of human immunodeficiency virus (HIV)- and murine leukemia virus (MuLV)-based vectors. Our studies reveal that the posttranscriptiona 1 effect of the WPRE can significantly stimulate the expression of luciferase and green fluorescent protein (GFP) in a promoter-indepe ndent fashion in both the retroviral and lentiviral systems (Zufferey et al., 1999). The ability of the WPRE to elevate transgene expression posttranscriptiona lly in the retroviral context is an indication that it may be useful in enhancing gene expression in the context of other potential gene therapy vectors. However, it is unclear that the results obtained in the retroviral vector system can be extrapolated to other gene delivery systems. Features that distinguish retroviruses generally, such as the presence of an RNA intermediate in the life cycle and the structure of the provirus, allow the possibility that the WPRE may enhance gene expression in this context by ameliorating the effects of inherent limitations of such a system. In this study, we wished to determine whether the WPRE could be used as a tool for the improvement of transgene expression more generally. Adeno-associate d virus (AAV) is attractive as a gene therapeutic delivery system (Carter, 1992; Kotin, 1994; Flotte and Carter, 1995) and therefore we chose to test the effect of the WPRE in the context of a recombinant AAV (rAAV) vector. This human parvovirus can transduce a wide range of host cells, including nondividing cells, and can yield stable integrants, allowing long-term expression of transgenes (Cheung et al., 1980; Laughlin et al., 1986; McLaughlin et al., 1988; Samulski et al., 1989; Flotte et al., 1993; Xiao et al., 1996, 1997; Murphy et al., 1997; Snyder et al., 1997.) We find that the WPRE can stimulate gene expression in the context of transient and stable transfection of 293 cells and rAAV transduction of transform ed cells and primary cells. Out results indicate that the strategy of enhancing gene expression posttranscriptionally with the WPRE is broadly applicable, and that multiple gene delivery systems will benefit from its inclusion.

\section{MATERIALS AND METHODS}

\section{Construction of eGFP plasmids}

Plasmids used in transfection and transduction experiments were based on the eGFP-C1 vector (Clontech, Palo Alto, CA). Since the vector was originally intended for the construction of enhanced GFP (eGFP) fusion proteins, a triple-frame stop codon was inserted after the GFP reading frame to allow the use of the multiple cloning site for the addition of untranslated elements. The triple stop was inserted as a pair of annealed synthetic oligonucleotides of sequences gatcttagctaactg (sense) and aatcagttagctaa (antisense) with BglII and EcoRI ends into the eGFP-C1 vector to produce the plasmid eGFP.stop. Bases 900-1750 of the WPRE were amplified by polymerase chain reaction (PCR) from the viral DNA template of WHV8 (accession number J04514), to yield a product with ClaI restriction sites at each end. This fragment was cut with ClaI and inserted into the eGFP.stop vector at the AccI site, to produce the plasmid eGFP.stop.WPRE.

To adapt the constructs for use in AAV, the plasmid eGFP.stop was cut with $S s p$ I to remove the f1 ori and amp promoter and religated, reducing the size of the recombinant AAV genome eventually generated and producing plasmid eGFP.stop. $\Delta$ Ssp. To allow transfer of the expression cassettes to the AAV shuttle vector psub201 (Samulski et al., 1987), synthetic linkers consisting of annealed oligonucleotides contain-

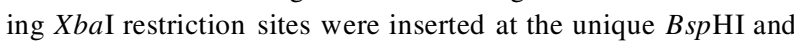
Ase I sites in eGFP.stop. $\Delta$ Ssp, yielding eGFP.stop'. The WPREbearing derivative was constructed as before by insertion of the ClaI-digested PCR product at the AccI site. The expression cassettes with and without WPRE were then transferred into the XbaI site of the AAV shuttle vector psub201, resulting in pAAV.eGFP.W and psub201.eGFP.stop. To generate pAAV.eGFP.W(as), bases 900-1750 of WHV8 were amplified by PCR to yield a product with AseI restriction sites at each end. The PCR product was cut with AseI and inserted in the antisense orientation into the AseI site of the psub201.eGFP.stop vector. All final plasmid products were grown in Escherichia coli HB101 and isolated by cesium chloride density gradient centrifugation.

\section{Tissue culture and transfections}

293 and HSF8 cells were grown at $37^{\circ} \mathrm{C}\left(5 \% \mathrm{CO}_{2}\right)$ in Dulbecco's modified Eagle's medium supplemented with $10 \%$ fetal calf serum (FCS). 293 cells were transfected at $30 \%$ confluency by the calcium phosphate method. The medium was changed after $18 \mathrm{hr}$. Cells transfected transiently were harvested after $48 \mathrm{hr}$. When stable selection was required, geneticin (G418, $200 \mu \mathrm{g} / \mathrm{ml}$; GIBCO, Grand Island, NY) was added to the medium. Pools of stably transfected cells were analyzed after 4 weeks of selection unless otherwise indicated. For transient transfections, molar equivalents of eGFP.stop $(0.85 \mu \mathrm{g})$ or eGFP.stop.WPRE ( $1 \mu \mathrm{g})$ were employed. To ascertain whether any difference in expression was due to a difference 
in transfection efficiency, the plasmids were cotransfected with $19 \mu \mathrm{g}$ of pCMXCD4, a cytomegalovirus (CMV) promoterdriven CD4 expression vector. Additional pUC118 was included to equalize the total amount of DNA present in each case. Mock and control transfections using pCMXCD4 or eGFP.stop.WPRE and pUC118 were equalized for total amounts of DNA, and were used to set the quadrant gates during flow cytometry.

\section{Preparation of $A A V$ stocks and transductions}

Purified viral stocks were generated by cotransfecting the plasmids pAAV.eGFP.W(as) and pAAV.eGFP.W into 293 cells with a Rep-Cap expression plasmid. Helper activities were supplied using a plasmid cotransfection-bas ed system as described (Xiao et al., 1998). Sixty hours after transfection, cells were lysed and the lysates fractionated twice by density centrifugation in cesium chloride (Fisher et al., 1996). Purified viral stocks were titered by dot-blot hybridization to a nitrocellulose membrane and comparison with known amounts of the starting plasmids; visualization was achieved by hybridization with a ${ }^{32} \mathrm{P}$ labeled GFP probe. Intensities were quantitated with a PhosphorImager (Molecular Dynamics, Sunnyvale, CA). The degree of wild-type AAV was determined by infectious center assays and in all preparations was less than $0.1 \%$. Cells were transduced in Dulbecco's modified Eagle's medium supplemented with $2 \%$ fetal calf serum. 293 cells were transduced at $30 \%$ confluency, HSF8 cells at $10 \%$. Viral stocks were added to the plates and the cells were incubated at $37^{\circ} \mathrm{C}\left(5 \% \mathrm{CO}_{2}\right)$ for $4 \mathrm{hr}$ with occasional agitation, after which the medium was supplemented with additional FCS to bring the serum content to $10 \%$. Cells were harvested for fluorescence-activ ated cell sorting (FACS) analysis after $48 \mathrm{hr}$ or after 4 weeks selection in $\mathrm{G} 418(200 \mu \mathrm{g} / \mathrm{ml})$.

\section{Flow cytometry}

293 or HSF8 cells were harvested and resuspended in phosphate-buffered saline (PBS) at approximately $10^{6}$ cells $/ \mathrm{ml}$. In all cases, except the analysis of transiently transfected 293 cells, in which an R-phycoerythrin-conjugated antibody was employed, propidium iodide was added to allow the exclusionary gating of dead cells. Where cells were stained for surface CD4, R-phycoerythrin -conjugated anti-CD4 antibody MT310 (Dako, Carpinteria, CA) was added to $10^{6}$ cells at a 1:100 dilution in $1 \mathrm{ml}$ of PBS-2\% FCS. After incubation for $30 \mathrm{~min}$ at $4^{\circ} \mathrm{C}$, the cells were washed twice with $15 \mathrm{ml}$ of cold PBS. All cells were analyzed for eGFP expression on a Becton Dickinson (San Jose, CA) FACScan. Within each experiment the samples were analyzed on the same day. Results are reported as geometric means. The geometric mean yielded values for fold increase that most closely matched those obtained by Western blotting when both assays were performed.

\section{RNA isolation and analysis}

Confluent cells from two $10-\mathrm{cm}$ plates were harvested by resuspension in PBS and brief centrifugation. Isolation of total RNA was accomplished by resuspending the cell pellet in $1 \mathrm{ml}$ of RNA STAT-50 LS (Tel-Test, Friendswood, TX). Nuclear and cytoplasmic RNA samples were prepared as previously de- scribed (Zufferey et al., 1999), except that the cytoplasmic lysis buffer consisted of $10 \mathrm{~m} M$ Tris ( $\mathrm{pH} 8.0$ ), $1.5 \mathrm{mM} \mathrm{MgCl} \mathrm{M}_{2}$, $140 \mathrm{mM} \mathrm{NaCl}, 20 \%$ glycerol, and $0.5 \%$ Nonidet P-40 (NP-40). RNA was fractionated on a $6 \%$ formaldehyde agarose gel and transferred to Duralon UV membranes (Stratagene, La Jolla, CA). Blots were hybridized in Quikhyb (Stratagene) with random primed, $\left[{ }^{32} \mathrm{P}\right]$ dCTP-labeled probes generated with the Prime-It II kit (Stratagene). The probe for eGFP was generated by digestion of eGFP-C1 with NdeI and PstI; the probe for the neomycin resistance gene was generated by digestion with AvrII. Fragments of 1131 and 1015 bp, respectively, were purified from agarose gels. Signals were visualized by autoradiography and quantitated using a Molecular Dynamics PhosphorImager. Message size was assessed by mobility relative to $18 \mathrm{~S}$ and $20 \mathrm{~S}$ ribosomal RNA.

\section{DNA isolation and analysis}

Two 10-cm plates of confluent 293 or HSF8 cells were harvested. Genomic DNA was prepared using the QiaAmp blood kit (Qiagen, Chatsworth, CA) according to the manufacturer instructions. Genomic DNA $(10 \mu \mathrm{g})$ was digested with PstI and NdeI for G418-selected lines transfected with eGFP-C1 derivatives. Digested DNA was fractionated on $0.8 \%$ agarose- $-0.5 \times$ TBE gels. After denaturation and neutralization, DNA was transferred to Duralon UV membranes (Stratagene) and hybridized with ${ }^{32} \mathrm{P}$-labeled probes. Signals were visualized by autoradiography and quantitated with a Molecular Dynamics PhosphorImager. Fragment size was assessed by comparison with a $\lambda H$ indIII/EcoRI digestion ladder.

\section{Western analysis}

293 cells were harvested and resuspended in Western lysis buffer (50 mM NaCl, $10 \mathrm{~m} M$ Tris [pH 7.5], 10\% glycerol, 1 $\mathrm{m} M$ dithiothreitol [DTT], 0.5\% NP-40), subjected to three cycles of freezing and thawing, and clarified by centrifugation. Proteins were normalized by the Bio-Rad (Hercules, CA) protein assay, and $50 \mu \mathrm{g}$ was fractionated by sodium dodecyl sulfate-polyacrylam ide gel electrophoresis (SDS-PAGE) on a $15 \%$ polyacrylamide gel. Proteins were transferred to PVDF-Plus membranes (Micron Separations, Westboro, MA) and incubated with a mouse anti-eGFP monoclonal antibody (Clontech), incubated with sheep anti-mouse ${ }^{125}$ I-conjugated antibodies. (Amersham Life Science, Arlington Heights, IL) and visualized by autoradiography.

\section{RESULTS}

\section{The WPRE increases GFP expression in the context of transient transfection}

To determine whether the WPRE could increase expression of GFP cDNA from vectors delivered by transfection, we employed derivatives of the eGFP-C1 vector (Clontech) either containing (eGFP.stop.WPRE) or lacking (eGFP.stop) the WPRE (Fig. 1A). GFP is detectable in intact cells by flow cytometry, allowing the assessment of variations in expression on a percell basis. In addition, GFP is already expressed well from this plasmid in the context of transfections, the message having been 

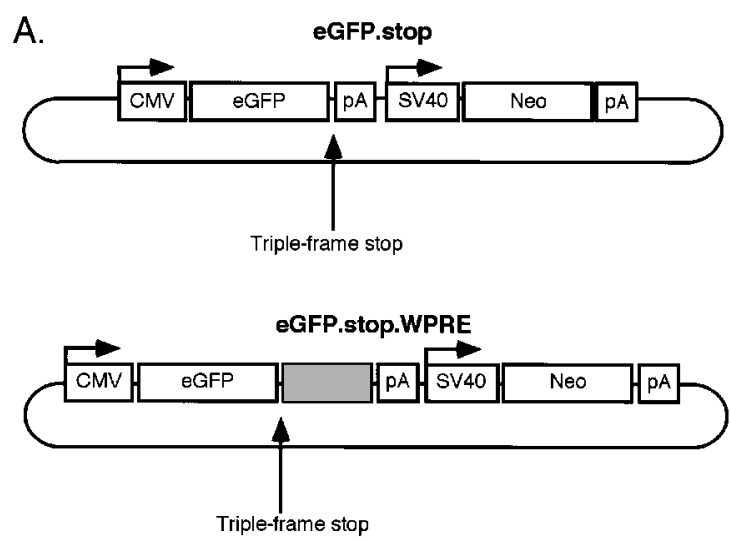

B.

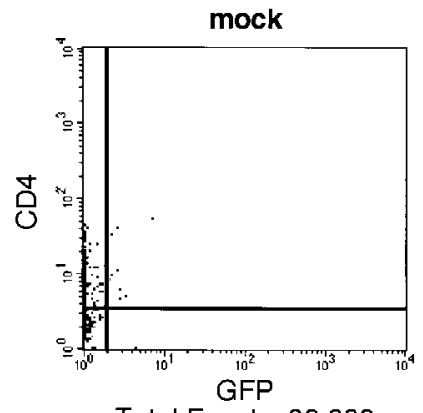

Total Events: 30,000

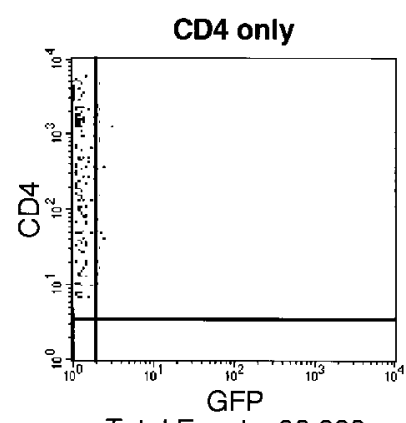

Total Events: 30,000

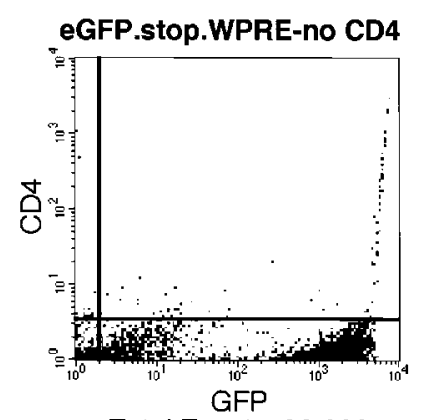

Total Events: 30,000

C.

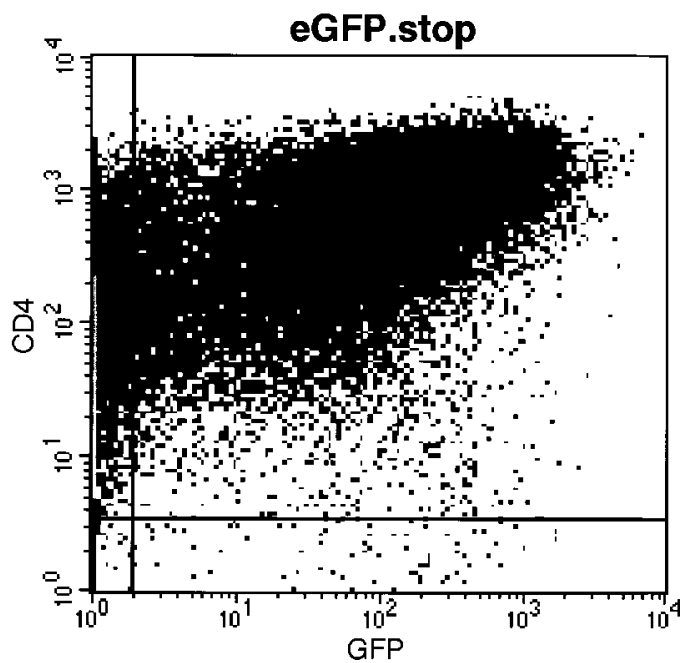

Total Events: 62885

Quad Events \% Gated X Geo Mean Y Geo Mean

$\begin{array}{lrrrr}\text { UL } & 32354 & 51.45 & 1.04 & 42.98 \\ \text { UR } & 29548 & 46.99 & 78.13 & 556.22 \\ \text { LL } & 880 & 1.40 & 1.01 & 2.05 \\ \text { LR } & 103 & 0.16 & 65.26 & 1.66\end{array}$

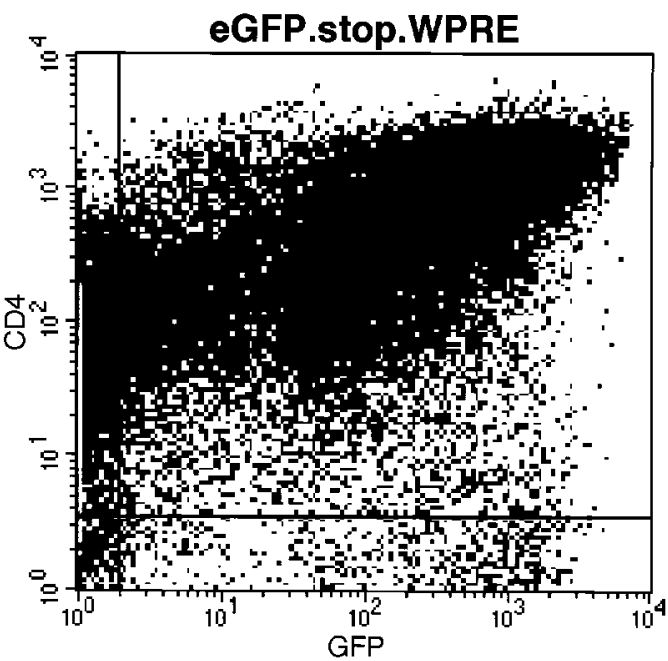

Total Events: 48162

Quad Events \% Gated X Geo Mean Y Geo Mean

$\begin{array}{lllll}\text { UL } & 16764 & 34.81 & 1.10 & 36.13\end{array}$

$\begin{array}{lllll}\text { UR } & 29525 & 61.30 & 121.21 & 394.68\end{array}$

$\begin{array}{lllll}\text { LL } & 974 & 2.02 & 1.07 & 1.89\end{array}$

FIG. 1. The WPRE enhances GFP expression in transiently transfected 293 cells. (A) GFP constructs used for transfection assays. (B and C) Flow cytometric analysis of 293 cells transiently transfected with eGFP.stop or eGFP.stop.WPRE. $x$ axis, GFP fluorescence; $y$ axis, anti-CD4 fluorescence. UL, UR, LL, and LR, upper left, upper right, lower left, and lower right quadrants, respectively; Geo Mean, geometric mean fluorescence. 
A.
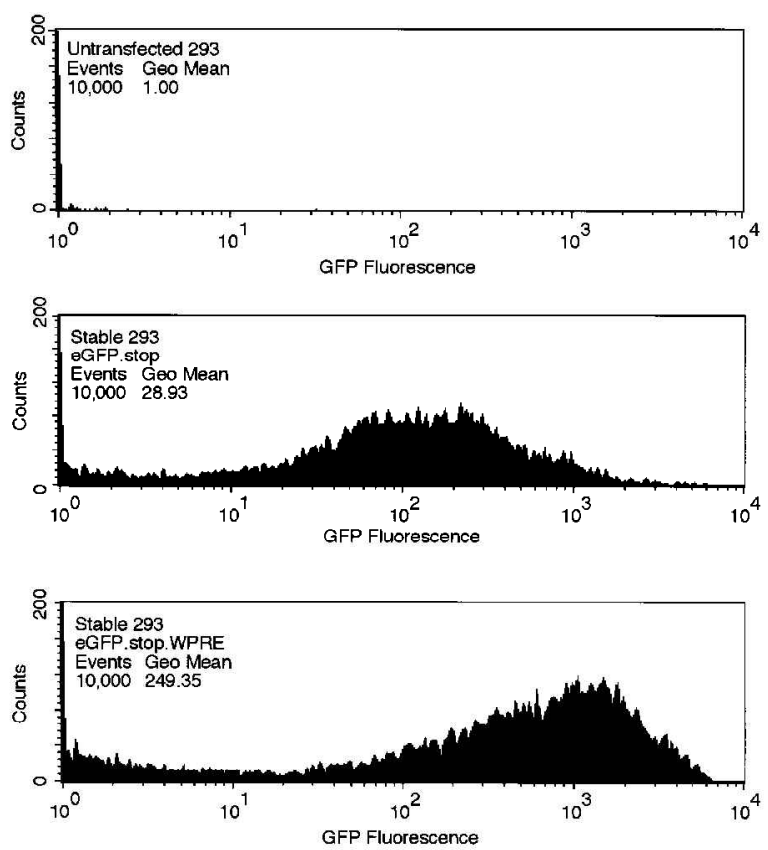

C.

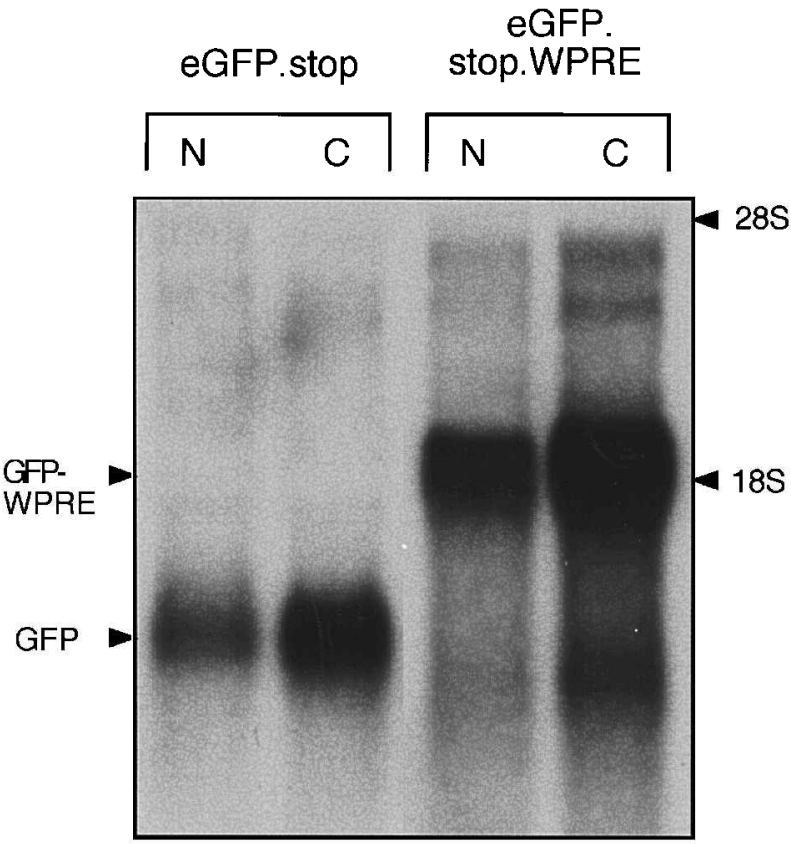

GAPDH

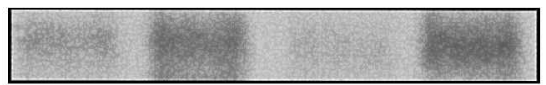

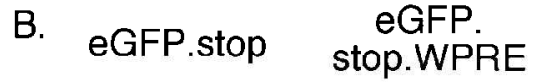

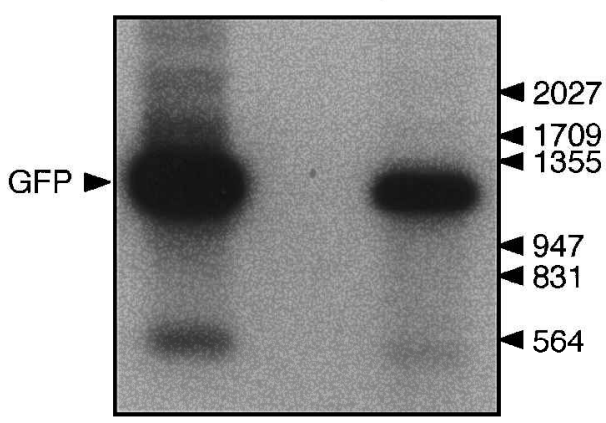

GAPDH

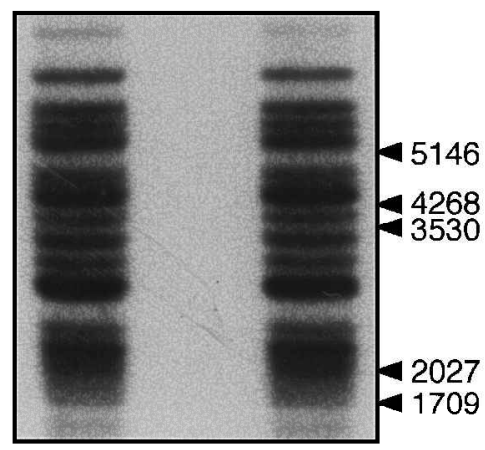

FIG. 2. The WHV PRE enhances transgene expression in 293 cells stably transfected with GFP-expressing constructs. (A) 293 cells were transfected with eGFP.stop or eGFP.stop.WPRE, and analyzed for GFP expression by flow cytometry. Geometric mean fluorescence (Geo Mean) is shown in the top left-hand corner. (B) Southern analysis of $20 \mu \mathrm{g}$ of genomic DNA from 293 cells stably transfected with plasmid eGFP.stop or eGFP.stop.WPRE. Blotting was done with GFP probe (top) or GAPDH probe (bottom). Positions of molecular weight standards are indicated. (C) The WPRE increases the level of transgene RNA in both the nuclear and cytoplasmic compartments of stably transfected 293 cells. Shown is a Northern analysis of nuclear and cytoplasmic RNA fractions prepared from 293 cells stably transfected with plasmid eGFP.stop oreGFP.stop.WPRE. Positions of the 18 and 28S ribosomal RNA species are indicated. optimized for codon usage and translation and the CMV promoter inducing high levels of expression. This made it an appropriate choice as a rigorous test of the ability of the WPRE to increase further the expression of an efficiently expressed transgene.

The ability of the WPRE to stimulate GFP expression in transient transfections was analyzed. eGFP.stop or eGFP.stop.WPRE was transfected into 293 cells by the calcium phosphate method. To control for efficiency of transfection, a CD4 expression plasmid was cotransfected in each case. At 48 $\mathrm{hr}$ the cells were harvested, stained for cell surface CD4, and subjected to flow cytometric analysis for CD4 and GFP ex- 
pression. The results indicated that, on a per-cell basis, levels of GFP expression increased significantly relative to those of CD4 expression when the WPRE was included in the GFP message (Fig. 1C). The controls showed less than $1 \%$ of the cell population in the upper right (UR) quadrant in all cases (Fig. 1B). Plotting of GFP fluorescence on the $x$ axis indicates a $50 \%$ increase in the geometric mean fluorescence (X Geo Mean, Fig. 1C) of the cell population in the UR quadrant, from 78 to 121 , when eGFP.stop.WPRE is used. No such increase is apparent on the $y$ axis, which represents the level of cotransfected CD4. Indeed, a slight decrease in CD4 was observed. It is also of note that the inclusion of the WPRE results in a $14 \%$ increase in the number of cells gated in the UR quadrant. The increase in GFP fluorescence in the presence of the WPRE-containing construct was therefore the result not of increased DNA uptake per cell, but of increased GFP expression per transfected plasmid. The results are representative of multiple experiments conducted with titrated amounts and multiple preparations of these plasmids.
The WPRE increases GFP expression in the context of stably transfected cell lines

The ability of the WPRE to stimulate GFP expression was analyzed when stable expression was selected with G418. The eGFP constructs contain the neomycin/kanamycin resistance gene driven by the simian virus 40 (SV40) promoter. 293 cells were transfected with eGFP.stop or eGFP.stop.WPRE, and selected for stable expression with G418 (GIBCO). The cell populations were then harvested and analyzed by flow cytometry. The geometric mean level of GFP expression in the G418-resistant cytometry. The geometric mean level of GFP expression in the G418-resistant populations was increased roughly ninefold as assessed by flow cytometry, from 29 to 249, when the WPRE was included (Fig. 2A).

Additional cells from the stably selected populations were harvested and subjected to Southern analysis, which indicated a fourfold greater content of GFP-encoding DNA in the case of eGFP.stop than of eGFP.stop.WPRE (Fig. 2B, top). Equal

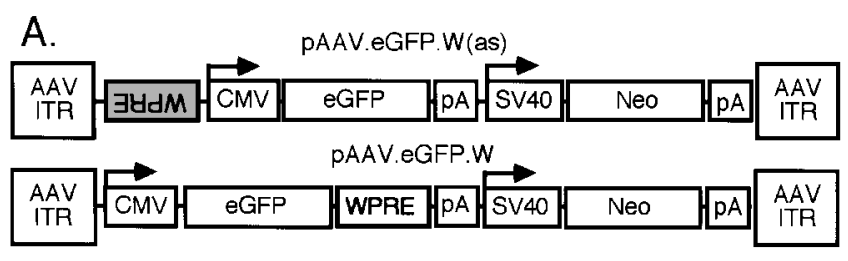

B.
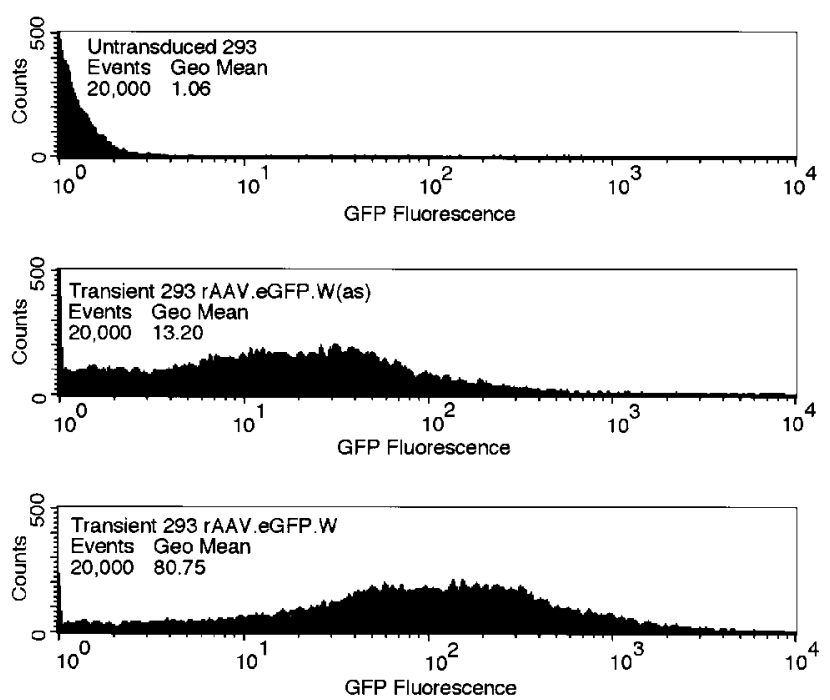

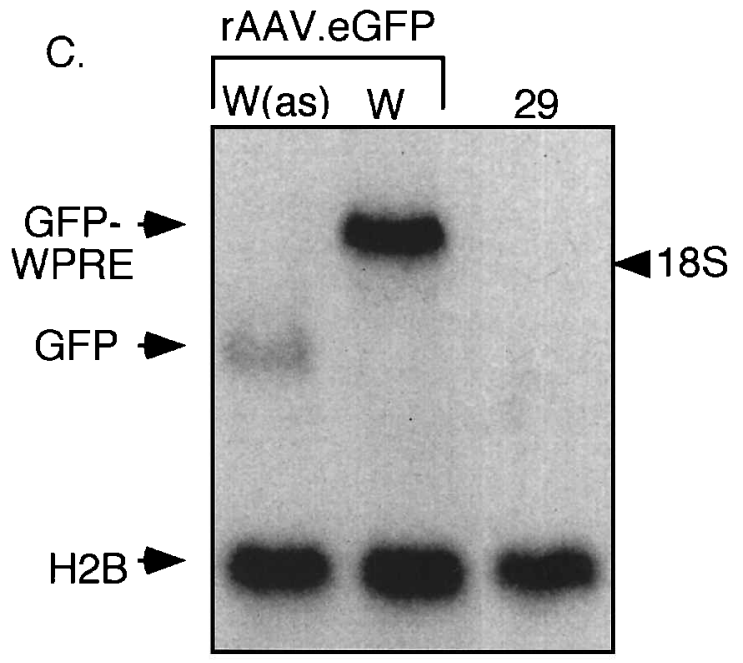

D. rAAV.eGFP

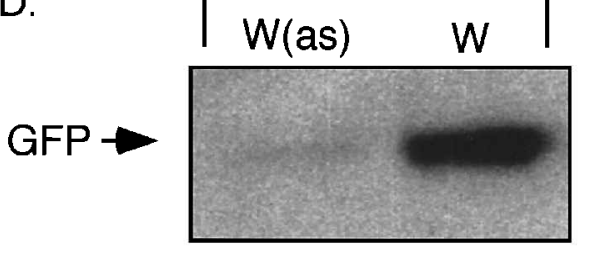

FIG. 3. The WHV PRE enhances transgene expression in 293 cells transiently transduced with recombinant AAV. (A) Constructs used in AAV viral stock preparation. (B) 293 cells transiently transduced with rAAV.eGFP.W(as) or rAAV.eGFP.W were analyzed for GFP expression by flow cytometry. Geometric mean fluorescence is shown in the top left-hand corner. (C) The WPRE increases the level of GFP RNA in transiently transduced 293 cells. Shown is a Northern analysis of $10 \mu \mathrm{g}$ of total cellular RNA from stably selected 293 cells transduced with rAAV.eGFP.W(as) or rAAV.eGFP.W. Position of the 18S ribosomal RNA species is indicated. (D) Western analysis for GFP in $50 \mu \mathrm{g}$ of protein extracted from 293 cells stably transduced with rAAV.eGFP.W(as) or rAAV.GFP.W. 
loading of genomic DNA was demonstrated by hybridizing with a glyceraldehyde-3-p hosphate dehydrogenase (GAPDH) probe (bottom). The decrease in copy number observed in the case of the WPRE-containing construct may be due to GFP toxicity (Tsien, 1998). This decreased amount of GFP-WPRE encoding

A.
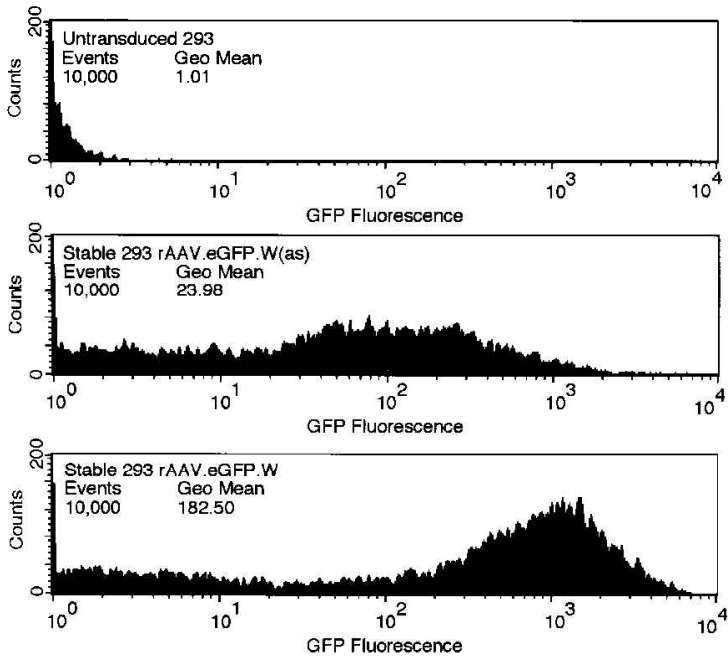

B.
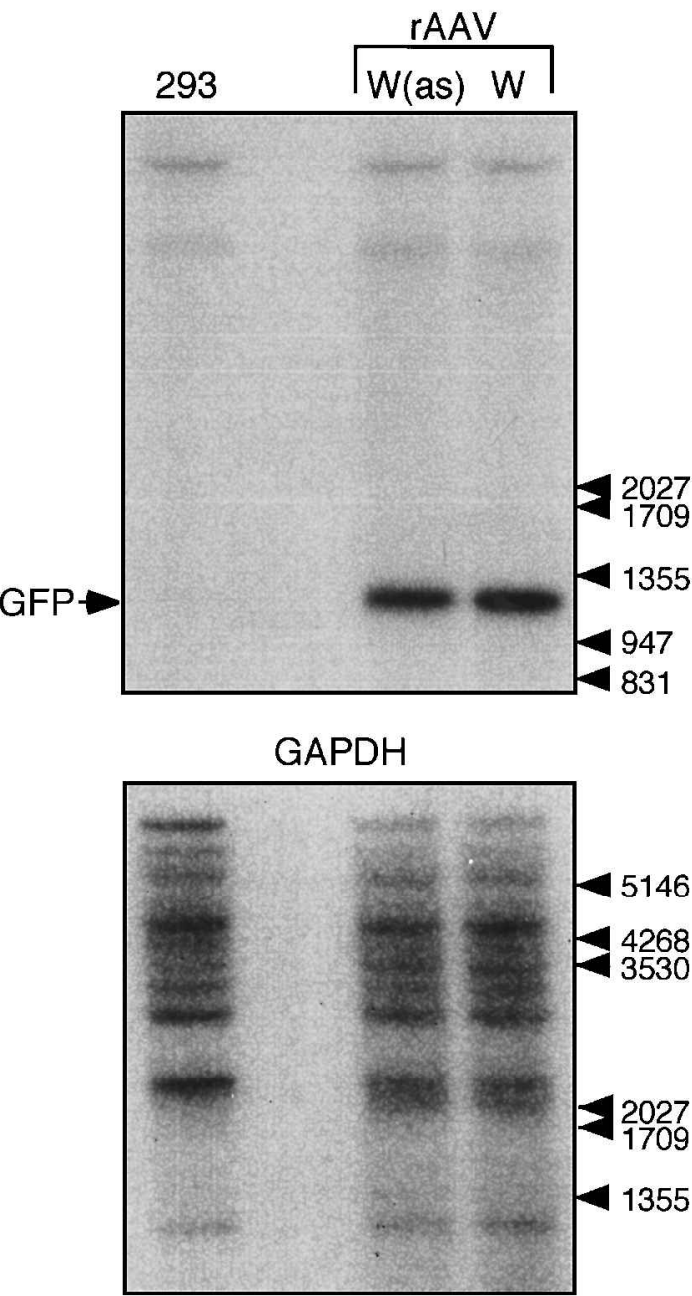

DNA after stable selection was observed in multiple independently derived cell populations. This inequality in GFP copy number, while not allowing direct normalization of GFP expression, reveals that the effect of the WPRE on expression is likely greater than is apparent in the flow cytometric analysis.

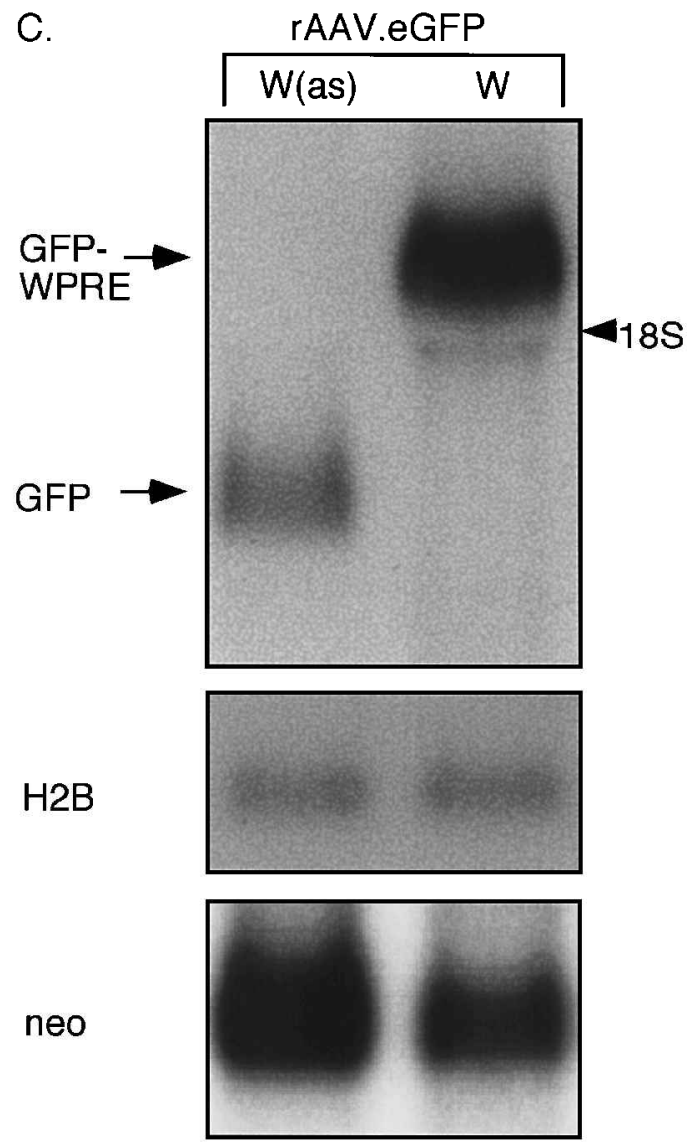

FIG. 4. The WHV PRE enhances transgene expression in 293 cells stably transduced with recombinant AAV. (A) 293 cells stably transduced with rAAV.eGFP.W(as) or rAAV.eGFP.W were analyzed for GFP expression by flow cytometry. Geometric mean fluorescence is shown in the top left-hand corner. (B) Southern analysis of genomic DNA from 293 cells stably transduced with rAAV.eGFP.W(as) or rAAV.eGFP.W. (C) The WPRE increases the level of transgene RNA in stable rAAV integrants. Shown is a Northern analysis of total cellular RNA from stably selected 293 cells transduced with rAAV. eGFP.W(as) or rAAV.eGFP.W. 
To characterize further the ability of the WPRE to enhance transgene expression in this context, RNA from the stably selected cells was subjected to Northern analysis. Inclusion of the WPRE caused a roughly fourfold increase in levels of GFPcontaining RNA in both the nuclear and cytoplasmic compartments of the cell (Fig. 2C, top). Probing with GAPDH demonstrated equal loading of RNA (bottom). These results indicate that, per vector copy, the WPRE-containing construct yielded significantly greater GFP RNA expression than its counterpart in both the nuclear and cytoplasmic compartments.

\section{The WPRE enhances the expression of GFP from genes delivered by adeno-associated viral transduction}

We next determined the potential of the WPRE as a tool for increasing transgene expression in the context of AAV vectors. The GFP and neo expression cassettes were inserted between AAV inverted terminal repeats (ITRs) in the plasmid psub201, resulting in the constructs shown in Fig. 3A. Since AAV genome packaging efficiency is dependent on the size of the insert bracketed by the ITRs (Dong et al., 1996), the WPRE was inserted in the antisense orientation upstream of the CMV promoter in construct rAAV.eGFP.W(as) both as a control for the presence of the WPRE and to promote equivalent packaging of the genomes in the two viruses. This configuration was used to avoid the inhibitory effect we had previously observed when the WPRE was cloned in the antisense orientation within the GFP transcript. This inhibition is believed to be caused by antisense transcripts derived from the WHV protein $X$ promoter, which is within the WPRE. To avoid any influence of the $\mathrm{X}$ promoter on GFP expression, the WPRE was cloned in the antisense orientation so that any transcripts derived from this promoter could not encode the reporter gene. From these constructs, purified viral stocks were generated and titrated. Titration determined that a multiplicity of infection (MOI) of 5000 viral genomes per target cell was sufficient to transduce more than $90 \%$ of the target cells under the experimental conditions (data not shown).

293 cells were transduced with rAAV.eGFP.W(as) and rAAV.eGFP.W, using an MOI of 5000 viral genomes per cell. After $48 \mathrm{hr}$ the cells were harvested, and subjected to flow cytometric analysis for GFP expression. Results representative of multiple experiments with multiple viral preparations indicate a sixfold increase in the geometric mean level of expression of GFP, from 13 to 81 , when the WPRE is included in the GFP message (Fig. 3B). Southern blotting of low molecular weight DNA extracted from transduced cells indicated similar viral uptake for both viruses (data not shown). To characterize this effect at the RNA level and to corroborate the quantitation of GFP by flow cytometry, the transiently transduced cells were harvested and subjected to Northern and Western analysis. Northern analysis of total cellular RNA, shown in Fig. 3C, indicated a threefold increase in GFP RNA. Amounts of GFP RNA were normalized relative to histone $2 \mathrm{~B}$ message detected by coprobing with an $\mathrm{H} 2 \mathrm{~B}$ fragment. This result demonstrates that the WPRE must be located within the GFP message to stimulate gene expression, suggesting that the WPRE functions at the posttranscriptional level in the AAV vector context. Western analysis of transduced cell lysates (Fig. 3D) showed a sevenfold increase in GFP protein in the presence of the WPRE, which corresponds well with the sixfold increase in GFP fluorescence as assessed by flow cytometry. The Northern and Western analyses exhibit different fold increases when the WPRE is included in the viral construct. This difference may be due to a translational effect of the WPRE.

To assay the effect of the WPRE on the expression of GFP in stably transduced cells, 293 cells were transduced with 5000 genomes per target cell of viruses rAAV.eGFP.W(as) and rAAV.eGFP.W, and placed under selection with G418. The stably transduced cells were subjected to flow cytometric, Southern, and Northern analyses. Figure 4A shows the results of flow cytometric analysis for GFP fluorescence. The effect of the WPRE, a roughly eightfold increase in geometric mean expression of GFP from 24 to 183, was similar to that observed in stably transfected cells. Southern analysis with a GFP probe indicated that the copy number of integrated, GFP-expressing AAV genomes in the different cell populations was roughly equivalent (Fig. 4B). No evidence of GFP toxicity was observed under these conditions. This is likely due to the low copy number present as a result of AAV transduction, on the order of one
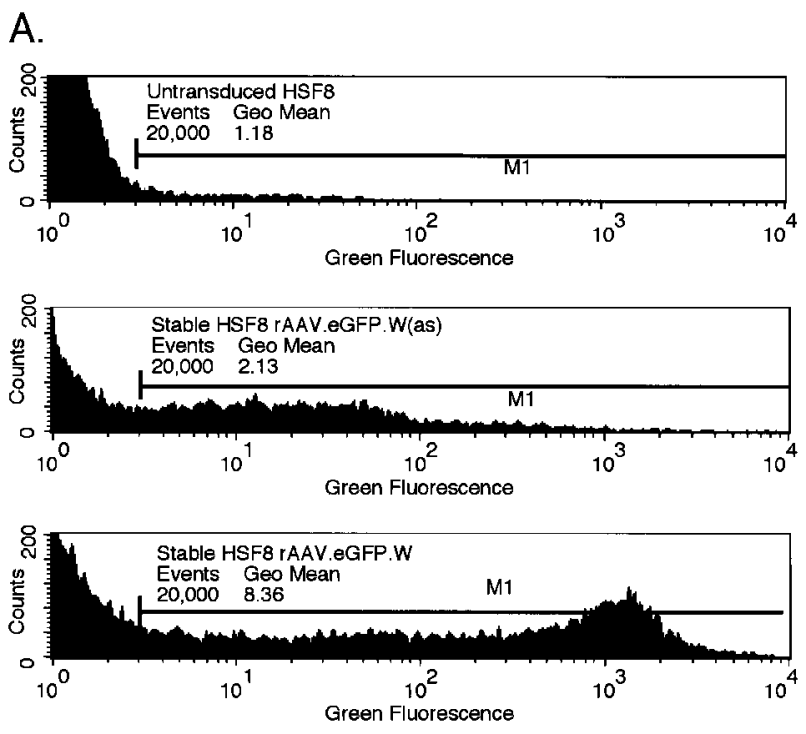

\section{B. rAAV.eGFP}

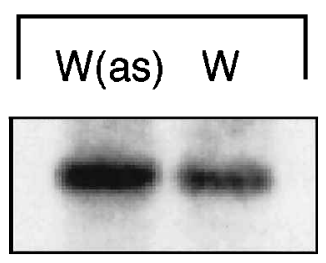

FIG. 5. The WPRE enhances GFP expression in primary human fibroblasts. (A) Primary human skin fibroblasts were stably transduced with rAAV.eGFP.W(as) or rAAV.eGFP.W and analyzed for GFP expression by flow cytometry. Geometric mean fluorescence is shown in the top left-hand corner. (B) Southern analysis of $10 \mu \mathrm{g}$ of genomic DNA from primary human skin fibroblasts stably transduced with rAAV.eGFP.W(as) or rAAV.eGFP.W. Blot was probed for GFP. GAPDH probing revealed equal loading of DNA (data not shown). 
to four, compared with the stably transfected cells, which could contain 10 or more copies of the GFP gene.

Northern analysis was performed on total cellular RNA isolated from the stably transduced populations. Probing with GFP and histone $2 \mathrm{~B}$ as a loading control indicated a fourfold increase in the amount of GFP message present in the cells transduced with rAAV.eGFP.W relative to those transduced with rAAV.eGFP.W(as) (Fig. 4C). Probing of this blot for neomycin/kanamycin RNA revealed that the increase in expression of GFP yielded by the WPRE is not related to an elevation of neomycin expression.

\section{The WPRE enhances GFP expression in primary human fibroblasts transduced with recombinant $A A V$}

The utility of the WPRE in a gene therapeutic context will be dependent on its ability to function in nontransformed human cells. To assess the plausibility of using the WPRE to enhance gene expression in such settings, 5000 genomes per target cell of viruses rAAV.eGFP.W(as) and rAAV.eGFP.W were used to transduce cultured primary human fibroblasts. The primary cells were acquired at a low passage number (passage 7) so as to allow ample time for selection and analysis. After 48 hr the cells were placed in selective medium (200 $\mu \mathrm{g}$ of G418 per milliliter). After 3 weeks of selection, the remaining cells were harvested and subjected to flow cytometric analysis for GFP expression. The results indicate a sixfold increase in the geometric mean level of expression of GFP when the WPRE is included in the GFP message (Fig. 5A). Southern analysis of genomic DNA, using a probe for GFP sequence, indicated that there is a slightly greater content of GFP-encoding DNA in the case of rAAV.eGFP.W(as) than of rAAV.eGFP.W (Fig. 5B). Reprobing for GAPDH revealed equal loading of genomic DNA (data not shown). These results are similar to those observed in 293 cells, and indicate that the WPRE can function to enhance gene expression in a heterologous viral context in cultured primary human cells.

\section{DISCUSSION}

Techniques currently used to introduce transgenes into target cells for gene therapy are based largely on methods evolved by viruses to deliver their genomes into host cells. The development of a new gene therapeutic vector frequently begins with a set of decisions regarding the best way to alter an existing viral genome; the virus must be made to contain the transgene of interest, incorporate it chromosomally or episomally into the desired cells, and allow it to be transcribed to a satisfactory level. While much effort has been spent optimizing gene therapeutic vectors for high titer, efficiency of entry, and sufficient levels of transcription, less progress has been made in the application of posttranscriptiona 1 methods for improving gene expression. New insight is rapidly being acquired into how viruses ensure high levels of expression of their genes at the posttranscriptional level. Multiple viruses have been shown to possess cis-and trans-acting elements whose purpose is to increase the expression of viral genes by interacting with host cell machinery (Malim et al., 1989; Fischer et al., 1994; Huang and Yen, 1994; Donello et al., 1996, 1998; Pasquinelli et al., 1997;
Saavedra et al., 1997). Previous work with the PREs has demonstrated their ability to rescue the expression of unspliced reporter messages (Donello et al., 1998), and of messages whose expression is dependent on the presence of introns (Huang and Yen, 1995) or posttranscriptiona 1 elements (Donello et al., 1998). This study and our previous work demonstrate that the WPRE can further increase the expression of a gene that is already well expressed. This suggests that enhancement can be expected to occur for a variety of potentially therapeutic genes, and will not be limited to genes that are difficult to express, although the benefit may be more dramatic in these cases.

We find that the WPRE is capable of enhancing the expression of GFP in the contexts of transient and stable transfection as well as in the context of DNA delivery by vectors derived from AAV. Only when the WPRE was located within a message did it have the ability to stimulate gene expression, demonstrating that the WPRE functions at the posttranscriptiona 1 level. It is possible that the WPRE contains an orientation-depend ent transcriptional enhancer that functions in AAV. However, we believe that this is unlikely since the WPRE region has been shown by several laboratories to have no detectable enhancer activity in a variety of contexts. Analysis of RNA in the nuclear and cytoplasmic compartments of stably transfected 293 cell populations revealed that the WPRE increased the amounts of message in both compartments. The nucleocytoplasm ic ratio relative to messages lacking the WPRE was not altered. In all cases, the increase in GFP protein expression mediated by the WPRE was approximately twofold greater than the observed increase in RNA, suggesting that the WPRE may also slightly stimulate the efficiency of translation. Together, these results suggest that the WPRE may have positive effects on gene expression in both the nuclear and cytoplasmic compartments of the cell. In addition, the WPRE is capable of enhancing the expression of GFP in primary human cells, establishing that its mechanism of enhancement is not dependent on the transform ed state of the cells. Inclusion of the WPRE is thus a viable means of increasing transgene expression in normal human tissue.

These results, together with our previous finding that the WPRE can stimulate transgene expression in the context of retroviral and lentiviral vectors, indicate that the WPRE will be a broadly useful tool for increasing levels of gene expression in multiple gene therapeutic and research settings. The effect of the WPRE on gene expression has been demonstrated in both RNA and DNA viruses, as well as in transfections with plasmid DNA. Thus, the increase in expression mediated by the WPRE is not attributable to the enhancement of an event in a specific viral life cycle. The WPRE can therefore be used in all gene delivery systems presently in clinical trial, as well as in vectors currently being designed and preclinically evaluated. The ability of the WPRE to increase the expression of each delivered DNA template will allow higher levels of transgene expression to be achieved per transduction event with currently available delivery systems. Such an increase in efficacy may permit the monetary cost of a course of gene therapy to be significantly reduced. Inclusion of the WPRE at the stage of vector design will permit a given therapeutic goal to be achieved with substantially less virus, thereby decreasing the cost of producing the reagents for therapy. The optimization of presently existing vectors may, as much as the design of new vectors, contribute to the arrival of gene therapy as clinical reality. The 
woodchuck hepatitis viral PRE will be a useful addition to nextgeneration gene delivery systems, which will incorporate measures to optimize gene expression at the posttranscriptional level.

\section{ACKNOWLEDGMENTS}

The authors gratefully acknowledge Allison Bocksruker for assistance in preparing this manuscript, John Donello and Dr. Romain Zufferey for helpful discussion, Lara Yurchak for the gift of the pCMXCD4 vector, and Dr. Wei Jiang for the gift of the primary fibroblasts. J.E.L. is a Chapman Scholar. M.D.W. is supported by the Oracle Foundation, by a gift from Odette Wurzburger, and by a grant from the President's Club of the Salk Institute. T.J.H. is supported by the Gene and Ruth Posner Foundation. This work was supported by Arthur and Larry Kramer. This work is dedicated to the memory of Jeffrey A. Posner.

\section{REFERENCES}

ANTONIOU, M., GERAGHTY, F., HURST, J., and GROSVELD, F. (1998). Efficient 3 '-end formation of human beta-globin mRNA in vivo requires sequences within the last intron but occurs independently of the splicing reaction. Nucleic Acids Res. 26, 721-729.

BUCHMAN, A.R., and BERG, P. (1988). Comparison of intron-dependent and intron-independe nt gene expression. Mol. Cell. Biol. 8, 4395-4405.

CARTER, B.J. (1992). Adeno-associated virus vectors. Curr. Opin. Biotechnol. 3, 533-539.

CHEUNG, A.K., HOGGAN, M.D., HAUSWIRTH, W.W., and BERNS, K.I. (1980). Integration of the adeno-associated virus genome into cellular DNA in latently infected human Detroit 6 cells. J. Virol. 33, 739-748.

CHOI, T., HUANG, M., GORMAN, C., and JAENISCH, R. (1991). A generic intron increases gene expression in transgenic mice. Mol. Cell. Biol. 11, 3070-3074.

DONELLO, J.E., BEECHE, A.A., SMITH, G.R., LUCERO, G.R., and HOPE, T.J., (1996). The hepatitis B virus posttranscription al regulatory element is composed of two subelements. J. Virol. 70, 4345-4351.

DONELLO, J.E., LOEB, J.E., and HOPE, T.J. (1998). Woodchuck hepatitis virus contains a tripartite posttranscriptional regulatory element. J. Virol. 72, 5085-5092.

DONG, J.Y., FAN, P.D., and FRIZZELL, R.A. (1996). Quantitative analysis of the packaging capacity of recombinant adeno-associated virus. Hum. Gene Ther. 7, 2101-2112.

FISCHER, U., MEYER, S., TEUFEL, M., HECKEL, C., LUHRMANN, R., and RAUTMANN, G. (1994). Evidence that HIV$1 \mathrm{Rev}$ directly promotes the nuclear export of unspliced RNA. EMBO J. 13, 4105-4112.

FISHER, K.J., GAO, G.P., WEITZMAN, M.D., DEMATTEO, R., BURDA, J.F., and WILSON, J.M. (1996). Transduction with recombinant adeno-associate $d$ virus for gene therapy is limited by leading-strand synthesis. J. Virol. 70, 520-532.

FLOTTE, T.R., and CARTER, B.J. (1995). Adeno-associated virus vectors for gene therapy. Gene Ther. 2, 357-362.

FLOTTE, T.R., AFIONE, S.A., CONRAD, C., McGRATH, S.A., SOLOW, R., OKA, H., ZEITLIN, P.L., GUGGINO, W.B., and CARTER, B.J. (1993). Stable in vivo expression of the cystic fibrosis transmembrane conductance regulator with an adeno-associated virus vector. Proc. Natl. Acad. Sci. U.S.A. 90, 10613-10617.
HARRIS, M.E., DONELlO, J.E., LOEB, J.E., OTERO, G.C., and HOPE, T.J. (1999). A cis-acting RNA element that increases expression by promoting longer polyadenylated tails. (In preparation.)

HUANG, Y., WIMLER, K.M., and CARMICHAEL, G.G. (1999). Intronless mRNA transport elements may affect multiple steps of premRNA processing. EMBO J. 18, 1642-1652.

HUANG, Z.M., and YEN, T.S. (1994). Hepatitis B virus RNA element that facilitates accumulation of surface gene transcripts in the cytoplasm. J. Virol. 68, 3193-3199.

HUANG, Z.M., and YEN, T.S. (1995). Role of the hepatitis B virus posttranscriptional regulatory element in export of intronless transcripts. Mol. Cell. Biol. 15, 3864-3869.

KOTIN, R.M. (1994). Prospects for the use of adeno-associated virus as a vector for human gene therapy. Hum. Gene Ther. 5, 793-801.

LAUGHLiN, C.A., CARDELliCHIO, C.B., and COON, H.C. (1986). Latent infection of $\mathrm{KB}$ cells with adeno-associated virus type 2. J. Virol. 60, 515-524.

MALIM, M.H., HAUBER, J., LE, S.Y., MAIZEL, J.V., and CULLEN, B.R. (1989). The HIV-1 Rev trans-activator acts through a structured target sequence to activate nuclear export of unspliced viral mRNA. Nature (London) 338, 254-257.

MCLAUGHLIN, S.K., COLLIS, P., HERMONAT, P.L., and MUZYCZKA, N. (1988). Adeno-associated virus general transduction vectors: Analysis of proviral structures. J. Virol. 62, 1963-1973.

MURPHY, J.E., ZHOU, S., GIESE, K., WILLIAMS, L.T., ESCOBEDO, J.A. and DWARKI, V.J. (1997). Long-term correction of obesity and diabetes in genetically obese mice by a single intramuscular injection of recombinant adeno-associated virus encoding mouse leptin. Proc. Natl. Acad. Sci. U.S.A. 94, 13921-13926.

NESIC, D., CHENG, J., and MAQUAT, L.E. (1993). Sequences within the last intron function in RNA 3 '-end formation in cultured cells. Mol. Cell. Biol. 13, 3359-3369.

PASQUINELLI, A.E., ERNST, R.K., LUND, E., GRIMM, C., ZAPP, M.L., REKOSH, D., HAMMARSKJOLD, M.L., and DAHLBERG, J.E. (1997). The constitutive transport element (CTE) of Mason-Pfizer monkey virus (MPMV) accesses a cellular mRNA export pathway. EMBO J. 16, 7500-7510.

RYU, W.S., and MERTZ, J.E. (1989). Simian virus 40 late transcripts lacking excisable intervening sequences are defective in both stability in the nucleus and transport to the cytoplasm. J. Virol. 63, 4386-4394.

SAAVEDRA, C., FELBER, B., and IZAURRALDE, E. (1997). The simian retrovirus- 1 constitutive transport element, unlike the HIV-1 RRE, uses factors required for cellular mRNA export. Curr. Biol. 7, 619-628.

SAMULSKI, R.J., CHANG, L.S., and SHENK, T. (1987). A recombinant plasmid from which an infectious adeno-associated virus genome can be excised in vitro and its use to study viral replication. J. Virol. 61, 3096-3101.

SAMULSKI, R.J., CHANG, L.S., and SHENK, T. (1989). Helper-free stocks of recombinant adeno-associate d viruses: Normal integration does not require viral gene expression. J. Virol. 63, 3822-3828.

SMITH, G.J., III, DONELlO, J.E., LÜCK, R., STEGER, G., and HOPE, T.J. (1998). The hepatitis B virus post-transcriptional regulatory element contains two conserved RNA stem-loops which are required for function. Nucleic Acids Res. 26, 4818-4827.

SNYDER, R.O., MIAO, C.H., PATIJN, G.A., SPRATT, S.K., DANOS, O., NAGY, D., GOWN, A.M., WINTHER, B., MEUSE, L., COHEN, L.K., THOMPSON, A.R., and KAY, M.A. (1997). Persistent and therapeutic concentrations of human factor IX in mice after hepatic gene transfer of recombinant AAV vectors. Nature Genet. 16, 270-276.

TSIEN, R. (1998). The green fluorescent protein. Annu. Rev. Biochem. 67, 509-544.

WANG, J.M., ZHENG, H., SUGAHARA, Y., TAN, J., YAO, S.N., 
OLSON, E., and KURACHI, K. (1996). Construction of human factor IX expression vectors in retroviral vector frames optimized for muscle cells. Hum. Gene Ther. 7, 1743-1756.

XIAO, X., LI, J., and SAMULSKI, R.J. (1996). Efficient long-term gene transfer into muscle tissue of immunocompe tent mice by adenoassociated virus vector. J. Virol. 70, 8098-8108.

XIAO, X., LI, J., MCCOWN, T.J., and SAMULSKI, R.J. (1997). Gene transfer by adeno-associate $\mathrm{d}$ virus vectors into the central nervous system. Exp. Neurol. 144, 113-24.

XIAO, X., LI. J., and SAMULSKI, R.J. (1998). Production of hightiter recombinant adeno-associated virus vectors in the absence of helper adenovirus. J. Virol. 72, 2224-2232.

ZUFFEREY, R., DONELLO, J.E., TRONO, D., and HOPE, T.J. (1999). Woodchuck hepatitis virus post-transcriptional regulatory el- ement (WPRE) enhances expression of transgenes delivered by retroviral vectors. J. Virol. 73, 2886-2892.

Address reprint requests to:

Dr. Thomas J. Hope

The Salk Institute for Biological Studies

10010 N. Torrey Pines Road

Infectious Disease Laboratory

P.O. Box 85800

La Jolla, CA 92037

E-mail: Hope@salk.edu

Received for publication March 15, 1999; accepted after revision June 29, 1999. 